\title{
Uma Arquitetura de Rede Neural Convolucional Simplificada para Reconhecimento da COVID-19 em Imagens de Raios-X
}

\author{
Fabio J Barbosa ${ }^{1}$, Alessandra M Coelho ${ }^{1}$, Matheus F O Baffa ${ }^{2}$ \\ ${ }^{1}$ Departamento Acadêmico da Ciência da Computação \\ Instituto Federal de Educação, Ciência e Tecnologia do Sudeste de Minas Gerais \\ campus Rio Pomba (IF Sudeste MG) \\ Rio Pomba - MG - Brasil \\ ${ }^{2}$ Departamento de Computação e Matemática \\ Universidade de São Paulo (USP) \\ Ribeirão Preto - SP - Brasil \\ fabiojbarbosa1@gmail.com
}

\begin{abstract}
COVID-19 is an emerging problem that has already caused great damage to society. An accurate diagnosis helps in providing more appropriate treatment for the patient. In the Computer Vision literature, many works implement robust deep learning architectures to create COVID classification models, this work aims to simplify a Convolutional Neural Network in order to reduce the computational cost, without losing performance in detecting COVID-19 in images of X-rays. With three layers of convolution, the work developed obtained an accuracy of $99.36 \%$ accuracy.
\end{abstract}

Resumo. A COVID-19 é um problema emergente que já causou grandes prejuízos à sociedade. Um diagnóstico preciso auxilia no encaminhamento do paciente a um tratamento mais adequado. Na literatura da Visão Computacional, muitos trabalhos implementam arquiteturas robustas de aprendizagem profunda para criação de modelos de classificação da COVID, este trabalho visa simplificar uma Rede Neural Convolucional de forma a reduzir o custo computacional, sem perder o desempenho na detecção da COVID-19 em imagens de Raios-X. Com três camadas de convolução, o trabalho desenvolvido obteve uma acurácia de 99,36\% de acurácia.

\section{Introdução}

Tendo a primeira aparição da COVID-19 em dezembro de 2019, na província Wuhan, na China, pouco tempo depois, em janeiro de 2020, já era considerada uma Emergência de Saúde Pública de Importância Internacional pela Organização Mundial da Saúde. No Brasil, a COVID-19 passou a ser considerada uma Emergência em Saúde Pública de Importância Nacional a partir de três de fevereiro de 2020 [Garcia and Duarte 2020].

A COVID-19 ou SARS-CoV-2, conhecida popularmente como Coronavírus, é causadora da síndrome respiratória aguda grave, pertencente à família Coronaviridae, da qual também são membros o SARS-CoV, vírus responsável pela síndrome respiratória aguda grave e o MERS-CoV , vírus responsável pela síndrome respiratória do Oriente Médio. Essas doenças podem causar problemas respiratórios graves e em alguns casos podem levar o paciente a morte [Lima 2020]. 
Além de testes laboratoriais e dos testes rápidos, o diagnóstico da COVID-19 também pode ser feito através da análise de imagens de Raios-X. Porém, pelo fato da COVID-19 ser uma doença nova, com sintomas comuns a outras síndromes gripais, os radiologistas têm certa dificuldade para o seu diagnóstico e diferenciação. Ainda, pelo fato das imagens radiográficas, em geral, terem baixo contrate, a leitura dos sinais da doença é dificultada [Meirelles 2020].

Por outro lado, a Visão Computacional propõe o desenvolvimento de sistemas inteligentes especializados na detecção de padrões nesse tipo de imagem [Martins et al. 2021]. Assim, é possível aplicar técnicas de Inteligência Artificial para auxiliar os médicos radiologistas na identificação de achados na imagem, de forma a promover um diagnóstico mais preciso.

Assim, objetiva-se neste trabalho o desenvolvimento de um método de classificação de imagens de Raios-X torácicas, utilizando uma Rede Neural Convolucional, com o intuito de auxiliar no diagnóstico de pacientes com o SARS-CoV-2. Desejase investigar também a possibilidade de simplificação da arquitetura de classificação da Rede Neural proposta, de forma a reduzir os custos computacionais, minimizando a perda de desempenho. A principal contribuição deste trabalho é a construção do modelo de classificação e a avaliação de diferentes arquiteturas baseadas em Redes Neurais Convolucionais, utilizando estruturas internas simplificadas.

\section{Materiais e Métodos}

\subsection{Base de Dados e Pré-Processamento}

A base de dados utilizada neste trabalho é originalmente composta por 6.432 imagens de radiografias do tórax, sendo 1.583 imagens de pacientes saudáveis, 576 imagens de pacientes diagnosticados com COVID-19 e 4.273 imagens de pacientes com pneumonia viral. A base é disponibilizada de forma gratuita e sendo formada por um conjunto de imagens de diferentes repositórios disponibilizada publicamente [Patel 2021]. Como este trabalho apresenta uma proposta de classificação binária, entre pacientes doentes (com COVID-19) e saudáveis, as imagens de pacientes com outros tipos de pneumonias virais não foram utilizadas. Além disso, a fim de balancear o número de amostras de cada classe disponível na base de dados, foram selecionadas aleatoriamente 500 imagens de pacientes saudáveis e 500 imagens de pacientes com COVID-19 para compor a base de dados final. O pré-processamento da base de dados consistiu em normalizar as imagens para escala de tons de cinza.

\subsection{Arquitetura da Rede Neural Convolucional}

Neste trabalho propomos a avaliação de arquiteturas simplificadas de Redes Neurais Convolucionais (Convolutional Neural Network - CNN) para detecção da COVID-19 em imagens de Radiografia do Tórax. As Redes Neurais Convolucionais foram escolhidas por proporcionar em sua arquitetura, estruturas que representam o conteúdo semântico da imagem utilizando filtros convolucionais (Feature Maps). Analisando o estado da arte, tal vetor descritivo tem se mostrado altamente eficiente em representar os pulmões para detecção de padrões entre as várias subarquiteturas de Redes Neurais Convolucionais, tais como a AlexNet, Visual Geometric Group (VGG) e a DenseNet [Rodrigues et al. 2020]. 
Assim, três arquiteturas de CNN foram desenvolvidas para a avaliação proposta. A primeira CNN (Modelo 1), foi configurada como se segue. A rede foi composta por quatro camadas, sendo uma camada de entrada, uma camada de convolução, uma camada densa e uma camada de saída. A camada de entrada foi responsável por receber a imagem no tamanho 256x256 pixels (convertido pela CNN) e transmitir os dados para as camadas à frente. Na camada de convolução foram extraídos 32 filtros de tamanho $3 x 3$ e foi utilizada a função de ativação Recifier Linear Unit (ReLU). Após a camada de convolução, realizou-se uma operação de MaxPooling, 2x2, a fim de realizar uma subamostragem da imagem. Em seguida, foi aplicada a operação de achatamento (Flatten), que teve como finalidade alterar o formato dos Feature Maps para o formato de um vetor unidimensional. Em seguida, adicionou-se uma camada de neurônios totalmente conectados com 1024 neurônios, com função de ativação ReLU, sendo a principal função dessa camada a detecção de padrões no vetor descritor construído pela convolução. Por fim, a camada de saída foi composta por um neurônio, responsável pela classificação binária, utilizando a função de ativação Sigmoid.

O Modelo 2 foi composto por dois blocos sequenciais de convolução e MaxPooling, extraindo 32 filtros cada de tamanho $3 \times 3$, e uma operação de achatamento com uma saída unitária, com ativação Sigmoid. De modo similar, o Modelo 3 foi composto por três blocos em sequenciais de convolução e MaxPooling, seguidos por uma operação de achatamento, com uma saída unitária. A Figura 1 contém um esquema representando a arquitetura das três CNN desenvolvidas. Podemos destacar que a principal diferença entre os modelos está no número de blocos, o Modelo $1 \mathrm{com}$ apenas um bloco, o Modelo 2 com dois blocos e, por fim, o Modelo 3 com três blocos.

Figura 1. Arquitetura da Rede Neural Convolucional proposta.

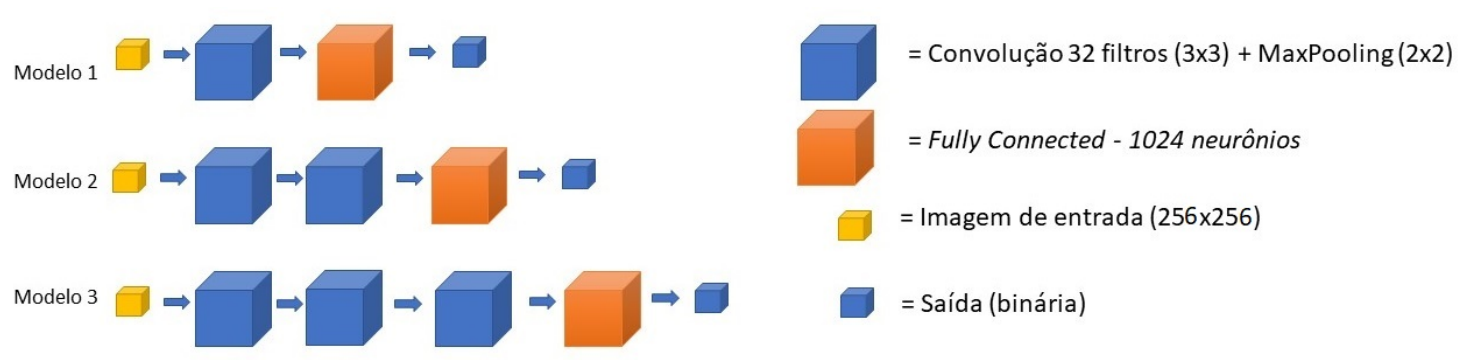

\section{Experimentos e Resultados}

Para a validação dos modelos de classificação gerados pelas CNNs, utilizou-se o algoritmo $K$-Fold Cross-Validation, para $k=5$. O $k$-fold divide a base em $k$ partes e realiza a mesma quantidade de experimentos, porém, a cada iteração, uma parte é utilizada para validação e o restante para treinamento. Por fim, calculou-se o resultado baseado na análise quantitativa, considerando a média das métricas ao longo dos cinco experimentos. As métricas quantitativas extraídas foram a acurácia, a sensibilidade, a especificidade, a área abaixo da curva ROC (Area Under the Curve - AUC) e a taxa de erro (Loss).

Ao realizar os treinamentos, percebeu-se que, ao aumentar o número de camadas, ocorria um aumento significativo no desempenho da rede. O Modelo 3, desenvolvido utilizando três blocos de convolução, obteve os melhores resultados quantitativos entre os modelos, produzindo uma acurácia média entre os $k$-folds de 99,36\%. 
Embora os resultados tenham sido levemente superiores, os Modelos 1 e 2 também apresentaram resultados excelentes, mostrando que modelos simples baseados em Redes Neurais Convolucionais podem realizar a tarefa de detecção de COVID-19 em radiografias do tórax de forma eficiente. Isso deve-se ao fato das características extraídas pela CNN serem altamente descritivas, sendo capaz de representar ambas as classes com clareza, do ponto de vista do algoritmo de detecção de padrões. A Tabela 1 contém os resultados obtidos no experimento realizado com cada modelo de CNN.

Tabela 1. Resultados dos testes dos modelos propostos.

\begin{tabular}{cccccc}
\hline Modelo & Acurácia(\%) & Sensibilidade(\%) & Especificidade $(\%)$ & AUC(\%) & Loss $(\%)$ \\
\hline Modelo 1 & 97,69 & 97,94 & 97,44 & 99,63 & 6,93 \\
Modelo 2 & 98,06 & 98,44 & 97,67 & 99,83 & 4,84 \\
Modelo 3 & 99,36 & 99,33 & 99,39 & 99,98 & 1,59 \\
\hline
\end{tabular}

\section{Conclusão}

A COVID-19 é uma doença recente que já causou grandes impactos na sociedade. O desenvolvimento de tecnologias de auxílio ao diagnóstico pode proporcionar ao paciente o encaminhamento de um tratamento mais adequado. Este trabalho propôs o desenvolvimento de uma Rede Neural Convolucional simplificada, criada a partir da avaliação de três arquiteturas distintas. Os resultados foram bem significativos, obtendo $99 \%$ de acurácia e $99 \%$ de sensibilidade. Em trabalhos futuros pretende-se, verificar a eficácia na classificação ao utilizar imagens de pneumonia viral, COVID-19 e saudáveis.

\section{Referências}

[Garcia and Duarte 2020] Garcia, L. P. and Duarte, E. (2020). Intervenções não farmacológicas para o enfrentamento à epidemia da COVID-19 no Brasil. Epidemiologia e Serviços de Saúde, 29.

[Lima 2020] Lima, C. M. A. d. O. (2020). Information about the new coronavirus disease (COVID-19). Radiologia Brasileira, 53:V - VI.

[Martins et al. 2021] Martins, J. V. G., Gregório, M. P., Baffa, M. d. F. O., and Coelho, A. M. (2021). Classificação da covid-19 em radiografias do tórax utilizando redes neurais profundas e padrões binários locais. Journal of Health Informatics, 12:387-392.

[Meirelles 2020] Meirelles, G. d. S. P. (2020). COVID-19: a brief update for radiologists. Radiologia Brasileira, 53:320-328.

[Patel 2021] Patel, P. (2021). Chest X-ray (Covid-19 \& Pneumonia). Disponível em: https://www.kaggle.com/prashant268/chest-xray-covid19-pneumonia. Acesso em: 01 jan. 2021.

[Rodrigues et al. 2020] Rodrigues, L., Rodrigues, L., da Silva, D., and Mari, J. F. (2020). Evaluating convolutional neural networks for covid-19 classification in chest x-ray images. In Anais do XVI Workshop de Visão Computacional, pages 52-57. SBC. 